\title{
Distributed Heterogeneous Database System Synchronization Problems in Intelligent Building
}

\author{
Hui Pang, Liting Gao, Fanxing Meng \\ Hebei Institute of Architecture Civil Engineering \\ Zhang Jia Kou, China
}

\author{
Bing Liu \\ NO.24 Middle School of Shi Jia Zhuang \\ Shi Jia Zhuang,China \\ pyanxuan@163.com
}

\begin{abstract}
Intelligent building is the product of the computer and information technology network to penetrate the construction industry, which fully reflects the architectural art and information technologies. This paper focuses on information platform in the intelligent building construction and application process for different sectors, different types of database's data integration, processing, capture data changes, transmission and synchronization. Through studied the method of data transformation, change data capture methods, making the integration of distributed heterogeneous databases in the process of data synchronization issues have been satisfactorily resolved.
\end{abstract}

Keywords-Intelligent building; Distributed heterogeneous database; Data synchronization

\section{INTRODUCTION}

With the development of science and technology, intelligent building research has become a hot topic in today's world, and an integrated intelligent building management system (IBMS) is the most important realization. The core technology of intelligent building system integration is to achieve the various integrated information exchange between subsystems of the integrated subsystems, to unified management and monitoring, it is very significance for integration of database and database synchronization. In the Intelligent Building, because the system's distribution and heterogeneous, leading to application-centric, every subsystem closed, poor scalability and interactivity, systems independent of each other, forming one islands of information. To overcome these problems, the entire intelligent building integration of information resources, the key is to achieve integration of heterogeneous databases. As little as possible impact on the local autonomy, Construct a global database of user needs to support the global application of the database and the heterogeneous databases of information exchange and sharing. Establish an effective central database to manage all the data in the distributed system is necessary. Data synchronization in distributed heterogeneous database, need to focus on solving the following problems: To make the different formats, heterogeneous database management systems can exchange data and conversion; capture change data in heterogeneous database; to maintain the autonomy of the existing system, ensuring the normal operation of the existing system to achieve synchronization of data between different databases.

\section{THE METHODS OF HETEROGENEOUS DATABASE INTEGRATION}

\section{A. Interface Technology (eg. ODBC)}

To facilitate data sharing among heterogeneous databases, Microsoft Specially made one for the database access application programming interface (API), the ODBC. To make the application operate different types of data sources, it is necessary to dynamically link to a different driver, the driver to complete the ODBC function calls and the exchange with the data source. This approach will involve a lot of code, reuse of data resources and transplantation are poor, lack of flexibility .It will increase the system's hardware and software costs, and the workload of the system maintenance will greatly increase.

\section{B. Use Dynamic SQL Statements}

Dynamic SQL statements are to use dynamic SQL statements to realize for all relational data processing. The processing method is to read the source data and store it to the variable, then insert to the table of the purpose library, to achieve data migration. Dynamic SQL statements are flexible, approach for a wide range, but complex, required to programming a visual interface. For the more common relational database, the amount of data smaller cases, you can use dynamic SQL statements for data conversion.

\section{The conversion technology based on XML and Web} services

XML's self-defining and scalability, based on the expression of various types of data, the expression of structured data independent of the platforms and the devices. It effectively implements the communication between computers. Meanwhile, with the popularity of WWW, almost all of the data is released by end-user's browser, so, XML inevitably become one of the most appropriate window between the back-end database to the outside world. XML and HTTP are the most basic protocol of the Web Services. HTTP has a good character ---through the firewall. $\mathrm{XML}$ is the basis of language for achieving the Web Services.

\section{THE METHOD OF CAPTURE CHANGE}

\section{A. Trigger Method}

In the source database, create insert trigger, delete trigger, and modify trigger for each synchronized source table. 
When the data is inserted, be deleted, or be modified into the source database, the trigger is activated. Use the trigger that records changes in the temporary table rows to get the change information of the source table. Restore the operations happened to SQL statement, and record the log file. Log file should contain the time of the operation, the SQL statement to be restored and other necessary information.

\section{B. Log Analysis Method}

Database log is an important tool for recovery data and maintain the integrity of the database. It already contains all the operating records of the information that submitted successfully. Log analysis method is by analyzing the log information of the database log to capture changes in sequence of synchronization objects. Because the database log already contains all the operating record that submitted successfully. As most of the database log's format does not open, need to use a dedicated log analysis tools or interface to parse the logical log of the database, to restore the operation happened to SQL statement and record the log files. Log files should contain the operation time, SQL statements, etc.

\section{Timestamp-based approach}

The method requires every table of the application systems in the event has a timestamp field to record the modification time of each table. This method does not affect the efficiency of the original application, but need to make larger adjustments to the original system, and can not capture the operation of the data changes that are not caused by the application itself.

\section{API method}

Some small non-relational database and the database haven't triggers and log mechanisms. Can be introduced a class of middleware between application and database, which will provide a range of API (including the ODBC driver), use the API to complete modifying the database by the application, at the same time to record the change sequence of synchronization objects.

\section{E. The method based on shadow table}

In many cases, the source database mustn't understand the each operation of the synchronized object, as long as know the final total happens is sufficient. So, during the time of initialization, make a shadow Table $S$ for synchronization object table T. Comparing the current contents of $\mathrm{T}$ and $\mathrm{S}$ to obtain the net change of the information.

\section{F. The method based on the triggers and log table}

The method of data capture based on the trigger and log table fits the real-time synchronization requirements to a certain extent. By the event-driven mechanism to take the initiative to calling for the Web services release by the Servers, and ultimately realize the database synchronization. This method is triggered the trigger during the operation, put the change information to the net change log table, followed according to the record information in the net change long table, by using the event-driven mechanism or manually for synchronization.

\section{INTELLIGENT BUILDING DATA SYNCHRONIZATION IMPLEMENTATION}

Resource management for the intelligent building of integrated information platform, mainly to property businesses in the Intelligent Building of the property equipment, property services, information dissemination and other daily property management resource integration, finally to form a unified information platform. The topology structure of the network is shown in Figure 1.

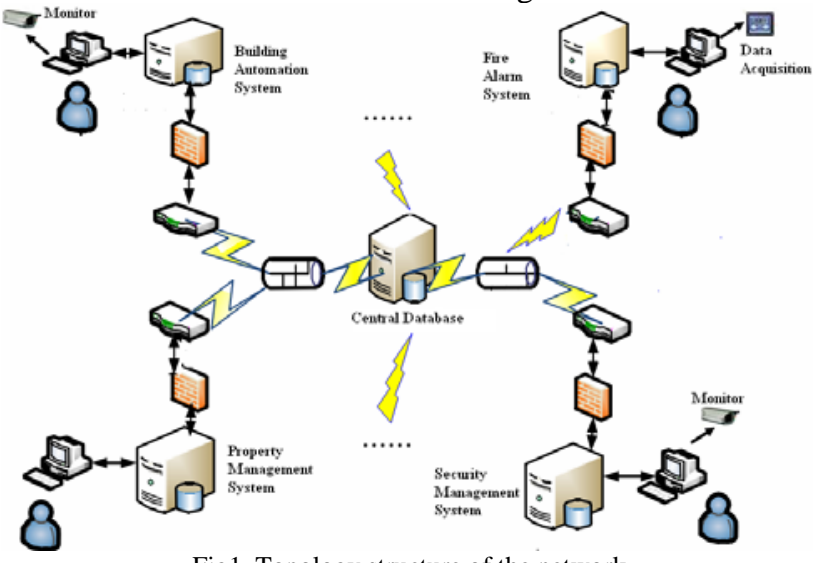

Fig1. Topology structure of the network

Currently, the core of the development of intelligent building technology is how to get the system data from multiple control systems, integrated in a unified control platform, to unify the decentralized management, to avoid the inefficient management and the data redundancy and other problem. The structure of the intelligent building management information platform is shown in Figure 2.

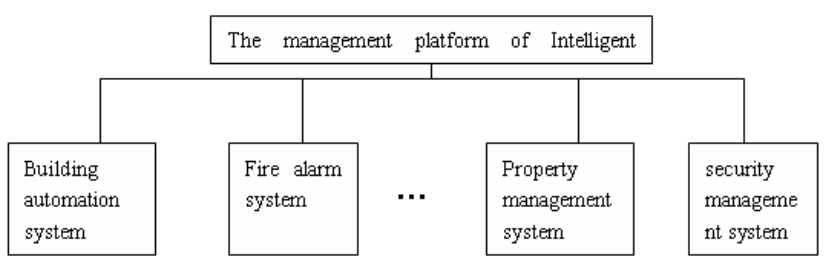

Fig.2 The structure of the intelligent building management information platform

In this paper, used based on the XML and Web services conversion technology, and based on triggers and the log methods to capture the data change, to realized data synchronization of the intelligent building heterogeneous database. The system can combined the control of smart systems integration and property management into a whole. While the corporate management of the subsidiary or branch office property management are also integrated in the regulatory platform. So it can improve the level of the management of the intelligent building and the management of enterprises themselves.

\section{A. The method used in heterogeneous database integration.}

Based on the heterogeneous database's local model to produce a global model-established a central database, 
integrated all the member database's shared data, to establish a unified authentication and access control mechanisms. The entire system structure is a star structure, in the center is a data exchange center can be used as servers. Data exchange center is surrounded by conversion module, which is the role of the adapter. Data conversion center server is data sharing and conversion centers. It used a standardized Web service interface for each node to provide data conversion services. Each data conversion modules of the data conversion node can interact with the convert platform by Web services. Each data conversion modules of the data conversion node can actively participate in data exchange with the agents of the heterogeneous source database information system. By use of data exchange tools, conversion module of the data conversion node to create a bi-directional mapping between the relational database and documents of the application and XML Schema of the share data to be published. We can call the central switching engine's Web services to register their own mode of ration. In order to achieve a unified data platform to solve the heterogeneity of the various subsystems' data and achieve data sharing and interoperability between subsystems, we use Web Services technology. Web Services use HTTP-based SOAP protocol. You can easily pass through the firewall. Using open standards can solve the problem of limited interoperability and improve the distributed computing capabilities. Web Services technologies, including many high-tech, but its core technology is mainly XML and SOAP. Although these two technologies also contain many elements, but its role can be simply summed up as, XML for data description, SOAP for data access. According to the role of these two technologies can be accurately derived using Web Services technology for multi-protocol system integration principles: first, the use of XML data describing to convert or map the information model of an automation equipment building, to form a kind of "self-contained and self-described" information model. Use SOAP's data access capabilities to access XML model, in order to achieve multi-protocol system of systems integration. Various subsystems of the intelligent building are actually the Web service provider. First, encapsulate these subsystems into a Web Services component, the service requester to provide a unified interface.

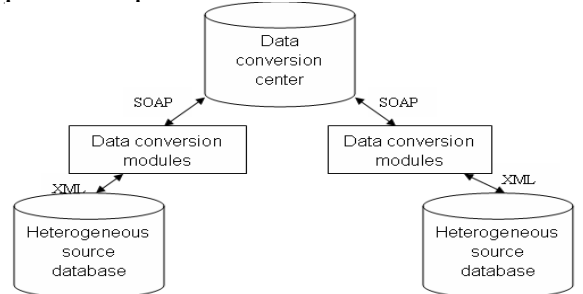

Fig.3 Data conversion model based on XML and Web Service

\section{B. The Method of Capture Change}

In the methods used to capture data changes is based on triggers and log. This method is triggered the trigger by the user during their operation, put the change information into the net change log table, then according to the information recorded in the net change log table, through the use of event-driven mechanism to synchronize. In data synchronization, first, source database use the components of the change capture to get the net change log table, and then through the event-driven transmission to realized data synchronization. Once synchronized, call the method of the center database's synchronized data in the logical processing. Synchronization is successful, get a success message from a central database, and then delete the net change in the log table. From the perspective of the central database, the central database use the components of the change capture to get the net change log table, and then through the event-driven transmission to realized data synchronization. Once synchronized, call the method of the source database's synchronizes data in the logical processing. Synchronization is successful, success message returned by the source database; delete the net change log table. The synchronization process is shown in Figure 4.

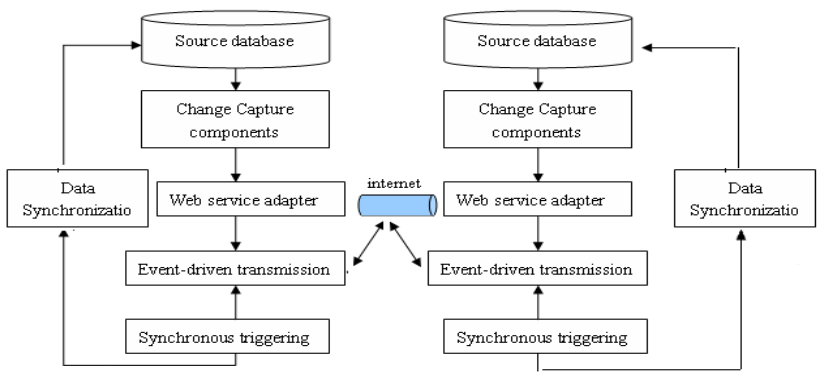

Fig.4 Float chats of data synchronization

\section{CONCLUSIONS}

This paper presents a of distributed heterogeneous database synchronization update model based on Web services. Applied the data capture methods of based on the trigger and log table. the use of heterogeneous database integration method based on Web services, the synchronization strategy based on Web services and event-driven transport mechanism can make the source database and the central database reliazed synchronization. This solves the intelligent building monitoring management procedures in the database synchronization problems.

\section{REFERENCES}

[1] Zhexin Pan, Lijuan Tong. Integration of distributed heterogeneous database based on Web Service.Application Research of Computers. 2006,23(3):208-210.

[2] Wei Liu, Lijuan Tong. Design about the method of Change Capture of Heterogeneous Database integration.Application Research of Computers. 2005,(7):213-215.

[3] Yushui Geng, Zhonghua Zhao, Xiangcui Kong. Synchronization System among Remote Heterogeneous Database Systems. Knowledge Acquisition and Modeling Workshop.2008:990-993.

[4] Chatterjee, N.Krishna, M.Semantic.Integration of Heterogeneous Databases on theWeb.Computing Theory and Applications. 2007:325-329.

[5] Min Shen, Huahu $\mathrm{Xu}$, Yonghua Ji. Distributed heterogeneous database system for data synchronization based on XML.Computer Engineering and Applications. 2005,5:184-186.

[6] Yujing Shi. Research on the data Transfer middleware between heterogeneous databases. Journal of Hebei University of Technology.2004.

[7] Yang Jiang. Research and implementation of Web Services-based data collection platform for Heterogeneous. Journal of Tianjin 
Proceedings of the 2012 2nd International Conference on Computer and Information Application (ICCIA 2012)

Polytechnic University.2007

[8] Yuanping Lei, Message mechanism to achieve synchronization of heterogeneous databases updated. Application Research of Computers. 2008,28(7):1700-1702.
[9] Peng Yang,Haitao Yang, Zhenghua Wang. Capture change and synchronization strategies of heterogeneous databases. Computer Engineering. 2008,34(16):53-55 